in Molprozenten gemacht werden, sind bei gleichen Konzentrationen in der Lösung des Stoffes mit höherem Polymerisationsgrad weniger Moleküle vorhanden als in einer mit niedrigerem Polymerisationsgrad. Für den Vorgang der Adsorption wird jedoch die Zahl der in Lösung vorhandenen kinetisch selbständigen Moleküle maßgebend sein. So ist, wie es auch die Versuchsergebnisse zeigen, eine größere Konzentration des höhermolekularen Zusatzes zur Erzielung der gleichen Beeinflussung notwendig als von einem weiter abgebauten Produkt. Diese Konzentrationsabhängigkeit ist dahingehend zu verstehen, daß die Methylcelluloseketten mit ihrer Molekülachse senkrecht zur Oberfläche des Quecksilbertropfens ad- sorbiert werden und so gewissermaßen eine „Molekelbürste “ bilden, deren „Borstenlänge “ vom Polymerisationsgrad abhängt. Würden nämlich die Methylcellulosemoleküle in ihrer ganzen Länge am Quecksilber adsorbiert werden, so dürfte unter der Annahme vergleichbarer Adsorptionskräfte keine Abhängigkeit vom Polymerisationsgrad zu erwarten sein und bei gleicher Gewichtskonzentration des Zusatzes auch gleiche Wirksamkeit gefunden werden müssen.

Für die praktische Polarographie ergibt sich daraus, $\mathrm{da}$ die höhermolekularen Methylcellulosen bis $\mathrm{zu}$ größeren Gehalten zwecks Maximaunterdrückung verwendbar sind, ohne daß dadurch eine Deformation der polarographischen Kurven eintritt.

\title{
Ebene Wellen endlicher Amplitude in idealen Gasen
}

\author{
Von Karl Bechert und Helmut Marx \\ Aus dem Institut für theoretische Physik der Universität Mainz \\ und den Optischen Werken E. Leitz, Wetzlar \\ (Z. Naturforschg. 6 a, 767-775 [1951]; eingegangen am 6. September 1951)
}

Der physikalische Zustand in einer ebenen Welle endlicher Amplitude läßt sich durch die Größen $v=\alpha c+u$ und $w=\alpha c-u$ beschreiben; $c$ ist die Schallgeschwindigkeit, $u$ die Strömungsgeschwindigkeit, $\alpha$ die Zahl der Freiheitsgrade der Gasmoleküle ( $(2)$. Häufig ist es bequemer, statt $v, w$ die Schallgeschwindigkeiten $c_{\mathrm{v}}, c_{\mathrm{w}}$ oder die Drucke $p_{\mathrm{v}}, p_{\mathrm{w}}$ zu verwenden, welche zu den beiden fortschreitenden Wellen gehören würden, die aus der $v$ - und $w$-Welle entstünden, wenn die $v$ - und $w$-Wellen sich einzeln in ungestörtem Gebiet ausbreiten könnten (§ 3). Mit diesen Größen lassen sich Vorgänge der Reflexion und der Rohrströmung besonders einfach beschreiben. Behandelt werden: Reflexion an einer festen Wand, Reflexion am offenen Ende eines Rohres, Reflexion an der Grenze von 2 Gebieten mit verschiedener Adiabatenkonstante, wie sie in Verbrennungsmotoren als die Gebiete des Verbrannten und Unverbrannten vorkommen $(\$ 4)$. Auch Druck und Strömungsgeschwindigkeit in einer beliebigen ebenen Welle können durch $p_{\mathrm{v}}, p_{\mathrm{w}}$ dargestellt werden. Wenn in einem Rohr mit einem offenen und einem geschlossenen Ende Überdruck erzeugt wird, so entstehen durch Reflexion der Wellen am offenen Ende Unterdruckwellen, die zum geschlossenen Ende zurücklaufen. Dieser Unterdruck wächst mit steigender Stärke der erzeugenden Überdruckwelle nur bis zu einem Grenzwert, der verschieden ist, je nach dem, ob das Gas aus Gebieten verschiedener Adiabatenkonstante besteht oder nicht (\$ 4). Der größte Unterdruck wird berechnet, der so bei der Reflexion an der festen Wand entsteht (\$ 4). Allgemeine Aussagen über die Strömung in einem solchen Rohr, wenn in der festen Wand Ventile angebracht sind, die aufgehen, sobald der Druck an dieser Wand unter den Außendruck sinkt (\$ 4). Wenn die Druckstörungen $\Delta p$ nicht wesentlich über das Doppelte des ungestörten Druckes $p_{\mathrm{n}}$ hinausgehen, steigen die zugehörigen $\Delta c$ nicht wesentlich über $0,1 c_{\mathrm{n}}$, wo $c_{\mathrm{n}}$ die ungestörte Schallgeschwindigkeit bedeutet. In diesem Gebiet kann man nach $\Delta c / c_{\mathrm{n}}$ entwickeln, während die akustische Näherung, welche Entwicklung nach $\Delta p / p_{\mathrm{n}}$ bedeutet, in diesem Gebiet versagt $(\$ 5)$. Die in $\$ 4$ abgeleiteten Beziehungen zwischen den $\Delta c_{\mathrm{i}} / c_{\mathrm{ni}}$ sind fast alle linear; es wird ein allgemeiner Satz bewiesen, wonach jede lineare $\mathrm{Be}-$ ziehung in den $\Delta c_{\mathrm{i}} / c_{\mathrm{ni}_{\mathrm{i}}}$ sich unmittelbar in eine näherungsweise gültige Produktbeziehung zwischen den zugehörigen „reduzierten Drucken“ $P_{\mathrm{i}}=p_{\mathrm{i}} / p_{\mathrm{n}}$ umschreiben läßt [Gl. (5.6), (5.8)]. Der Fehler gegenüber der strengen Gleichung ist von der Ordnung $\left(\Delta c_{\mathrm{i}} / c_{\mathrm{ni}}\right)^{2}$; er läßt sich leicht angeben bis zu Gliedern der Ordnung $\left(\Delta c_{\mathrm{i}} / c_{\mathrm{ni}}\right)^{3}$ einschließlich, (5.9). Anwendung auf die Beispiele von $\S 4$ zeigt, daß die Beziehungen zwischen den anschaulichen Größen $P_{\mathrm{v}}, P_{\mathrm{w}}, \mathrm{d} . \mathrm{h}$. zwischen den zugehörigen reduzierten Drucken der fortschreitenden Wellen, recht einfach werden (\$ 5). Auch die Strömungsgeschwindigkeit ist durch $P_{\mathrm{v}}, P_{\mathrm{w}}$ darstellbar (5.16). Man beherrscht so den in der Praxis wichtigen Bereich mäßig großer endlicher Amplituden mit einfachen Formeln. 


\section{\$1. Einleitung}

E bene Störungen in idealen Gasen können durch physikalische Größen beschrieben werden, die für fortschreitende Wellen kennzeichnend sind. Diese Betrachtungsweise ist besonders geeignet, das Wellenwandern in Motoren und in nicht zu langen Rohren zu beschreiben. Die Beschränkung auf „nicht zu lange Rohre" meint hier, daß wir Stoßwellen aus unserer Betrachtung ausschließen; wir betrachten auch nur solche Motorenvorgänge, bei denen Stoßwellen keine wesentliche Rolle spielen. Ein Beispiel dazu sind die Vorgänge im Schmidt-Rohr, dessen Theorie wir mit den Methoden der vorliegenden Arbeit in drei noch nicht veröffentlichten Arbeiten (1942/44) gegeben haben ${ }^{1}$.

Auch die Reibung und Wärmeleitung vernachlässigen wir, sie spielen nur bei sehr steilen Wellen und in besonders engen Rohren eine Rolle. Kommen auch Verbrennungsvorgänge neben dem Wellenwandern vor, so kann man häufig so schematisieren, daß das Gebiet des Verbrannten durch einen bestimmten Wert der Adiabatenkonstante charakterisiert wird, das Gebiet des Unverbrannten durch einen anderen. Die Verbrennungszone selbst, die Flamme, ist sehr dünn; ihre Dicke liegt in der Größenordnung von $10^{-2}$ bis $10^{-3} \mathrm{~cm}^{2}$. Interessiert man sich nur für das Wellenwandern, so wird man die Flamme als beliebig dünn schematisieren; auf jec̀en Fall braucht man über sie dann nur zu wissen, daß zu ihren beiden Seiten die Adiabatenkonstante einen bestimmten, auf beiden Seiten verschiedenen Wert hat.

\section{\$2. Die Grundgleichungen}

Wandergeschwindigkeit fester Werte von $v, w$

Die Wanderrichtung der Störungen sei die positive oder negative $x$-Richtung, wir bezeichnen die Dichte mit $\varrho$, die Strömungsgeschwindigkeit mit $u$, den Druck mit $p$, die Entropie pro g mit $S$. Dann lauten die Gleichungen der Erhaltung von Masse, Impuls und Energie:

$$
\begin{gathered}
\frac{\mathrm{d} \varrho}{\mathrm{d} t}+\varrho \frac{\partial u}{\partial x}=0 ; \\
\varrho \frac{\mathrm{d} u}{\mathrm{~d} t}+\frac{\partial p}{\partial x}=0, \\
\mathrm{~d} S \mathrm{~d} t=0 .
\end{gathered}
$$

$1 \mathrm{~K}$. B e chert u. H. Marx, Zur theoretischen Beschreibung der Vorgänge im Schmidt-Rohr.
Für ideale Gase ist:

$$
p=\varrho R T / M,
$$

wo $R$ die Gaskonstante, $T$ die absolute Temperatur und $M$ das Molekulargewicht bedeuten. Aus (2.3) folgt zunächst nur, daß die spezifische Entropie einer wandernden Gasschicht beim Weiterwandern erhalten bleibt, aber nicht, daß die Entropie überall im Gas denselben Wert habe. Wir machen aber jetzt die Annahme, daß die Entropie und damit die Adiabatenkonstante stückweise räumlich konstant sei. Bei Verbrennungsvorgängen z. B. soll sie im ganzen Gebiet des Verbrannten einen festen Wert haben, im ganzen Gebiet des Unverbrannten einen davon verschiedenen, aber auch räumlich überall gleichen Wert. Wir nehmen weiter an, daß das Verhältnis $\varkappa$ der spezifischen Wärmen praktisch temperaturunabhängig sei im betrachteten Temperaturbereich. An Stelle von $\%$ benützen wir meist die Größe $\alpha$, die durch die Gleichung definiert ist:

$$
\varkappa=1+\frac{2}{!} ;
$$

im Sinn der kinetischen Gastheorie ist $\alpha$ die Zahl der Freiheitsgrade des Gasmoleküls, liegt also bei Luft etwas über 5 .

Die Schallgeschwindigkeit $c$ ist definiert durch:

$$
\left(\frac{\partial p}{\partial \varrho}\right)_{S=\text { const }}=c^{2}
$$

Nennen wir das spezifische Volumen $s$, so gilt:

$$
S-S_{0}=c_{s} \lg \frac{p s^{*}}{p_{0} s_{0}^{*}}
$$

dabei ist $c_{\mathrm{s}}$ die spezifische Wärme bei konstantem Volumen. Für die „Adiabatenkonstante“ führen wir die Abkürzung $f$ ein:

$$
p_{0} s_{0}^{*}=f
$$

nach unseren Annahmen ist $f$ räumlich gebietweise konstant. Es ist:

$$
c^{2}=x f \varrho^{x-1} ; \quad \varrho=\left(\begin{array}{c}
c^{2} \\
\% f
\end{array}\right)^{1 /(x-1)} .
$$

Mit der Abkürzung:

wird:

$$
g=\frac{1}{\%}\left(\frac{1}{\varkappa f}\right)^{1 /(\%-1)}
$$

$$
p=g c^{a+2}
$$

auch $g$ ist räumlich gebietweise konstant.

2 K. B e chert, Z. Naturforschg. 3a, 584 [1948]: Ann. Physik (6) 4, 191 [1949]; 5, 349; 7, 113 [1950]: Z. Elektrochem. angew. physik. Chem. 54, 239 [1950]. 
Wir führen die Größen $v, w$ ein durch die Gleichungen:

$$
a c+u=v ; \quad a c-u=w,
$$

woraus folgt: $2 u=v-w ; 2 \alpha c=v+w$.

Für $v, w$ gelten wegen (2.1), (2.2) die Gleichungen:

$$
\frac{\mathrm{d} v}{\mathrm{~d} t}+c \frac{\partial v}{\partial x}=0 ; \quad \frac{\mathrm{d} w}{\mathrm{~d} t}-c \frac{\partial w}{\partial x}=0,
$$

oder auch:

$$
\frac{\partial v}{\partial t}+(u+c) \frac{\partial v}{\partial x}=0 ; \quad \frac{\partial w}{\partial t}+(u-c) \frac{\partial w}{\partial x}=0 .
$$

Die letzten 2 Gleichungen lehren, daß ein fester Wert von $v$ mit der Geschwindigkeit $u+c$ wandert, ein fester Wert von $w$ mit der Geschwindigkeit $u-c$. Wir schreiben dies:

$$
\dot{x}_{\mathrm{v}}=u+c ; \quad \dot{x}_{\mathrm{w}}=u-c .
$$

Stets ist die Relativgeschwindigkeit der $v$ - und der $w$-Welle gleich $2 c$. Solange $|u|<c$, ist $\dot{x}_{\mathrm{v}}>0$, $\dot{x}_{\mathrm{w}}<0$; die $v$-Welle wandert nach rechts, die $w$-Welle nach links. Wenn $|u|>c$, wandern beide Wellen in Richtung der Strömung $u$, bei positivem $u$ nach rechts, bei negativem $u$ nach links. Im ersten Fall ist die $v$-Welle die schnellere: $\dot{x}_{\mathrm{v}}>\dot{x}_{\mathrm{W}}$; im zweiten Fall die $w$-Welle. Wenn die Wellen sich frei ausbreiten können, trennen sich $v$ - und $w$-Welle voneinander, weil sie die Relativgeschwindigkeit $2 c$ haben.

\section{§ 3. Fortschreitende Wellen}

Die Größ e n $\Delta c_{\mathrm{v}}, \Delta c_{\mathrm{w}}$

Als „Normalzustand“ bezeichnen wir einen solchen, bei dem der Druck den Wert $p_{\mathrm{n}}$ hat und die Strömungsgeschwindigkeit Null ist. Auch an andere Größen hängen wir den Index $n$, wenn sie sich auf den Normalzustand beziehen. Haben wir verschiedene Gebiete i mit verschiedenen Adiabatenkonstanten $g_{i}$, so gilt:

$p_{\mathrm{n}}=g_{\mathrm{i}} c_{\mathrm{ni}}{ }^{a_{\mathrm{i}}+2} ; \quad u_{\mathrm{n}}=0 ; \quad v_{\mathrm{ni}}=w_{\mathrm{ni}}=a_{\mathrm{i}} c_{\mathrm{ni}}$.

Aus der Kenntnis von $\alpha_{\mathrm{i}}, g_{\mathrm{i}}$ und dem festgesetzten Wert von $p_{\mathrm{n}}$ können $c_{\mathrm{ni}}, v_{\mathrm{ni}}, w_{\mathrm{ni}}$ berechnet werden.

Allgemein ist für beliebigen Druck:

$$
p_{\mathrm{i}}=g_{\mathrm{i}} c_{\mathrm{i}}^{a_{\mathrm{i}}+2} \text {. }
$$

Wir nennen eine Welle „fortschreitende v-Welle“, wenn in ihr überall im betrachteten Gebiet $w$ den ungestörten Wert $w_{\text {ni }}$ hat; den zugehörigen $c$-Wert nennen wir $c_{\mathrm{vi}}$. Nach (2.12) ist:

$$
v_{\mathrm{i}}=2 \alpha_{\mathrm{i}} c_{\mathrm{vi}}-w_{\mathrm{ni}}=a_{\mathrm{i}}\left(2 c_{\mathrm{vi}}-c_{\mathrm{ni}}\right) .
$$

Gibt man andererseits $v_{\mathrm{i}}$ für eine beliebige Welle vor, so findet man aus (3.3) den Wert $c_{\mathrm{vi}}$, der als $c$-Wert zu diesem $v_{\text {i }}$ gehören würde, wenn $w_{\text {i }}$ gleich $w_{\text {ni }}$ wäre, wenn $v_{\text {i }}$ also eine fortschreitende $v$-Welle wäre. Man kann so $v_{\mathrm{i}}$ charakterisieren durch denjenigen Wert $c_{\mathrm{vi}}$ der Schallgeschwindigkeit, der einer fortschreitenden $v$-Welle entsprechen würde.

Dieselbe Überlegung läßt sich für $w$ machen. Eine Welle heißt "fortschreitende $w$-Welle", wenn in ihr überall im betrachteten Gebiet $v$ den ungestörten Wert $v_{\text {ni }}$ hat; der zugehörige $c$-Wert heißt $c_{\text {wi }}$. Es gilt:

$$
w_{\mathrm{i}}=2 \alpha_{\mathrm{i}} c_{\mathrm{wi}}-w_{\mathrm{ni}}=c_{\mathrm{i}}\left(2 c_{\mathrm{wi}}-c_{\mathrm{ni}}\right) .
$$

Ist $w_{\mathrm{i}}$ für eine beliebige Welle gegeben, so findet man aus (3.4) den Wert $c_{\text {wi }}$, der als $c$-Wert zu diesem $w_{\text {i }}$ gehören würde, wenn $w_{\text {i }}$ eine fortschreitende $w$ Welle wäre. Man kann so $w_{\mathrm{i}}$ charakterisieren durch denjenigen Wert $c_{\mathrm{wi}}$ der Schallgeschwindigkeit, der einer fortschreitenden $w$-Welle entsprechen würde.

Die Bezeichnung "fortschreitende Welle" rechtfertigt sich dadurch, daß z. B. $w=$ const eine $v$-Welle gleichbleibender Höhe und Breite bedeutet, die im Grenzfall der Akustik unverzerrt wandert.

Wir bilden die Größen:

$\left.\Delta v_{\mathrm{i}} \equiv v_{\mathrm{i}}-v_{\mathrm{ni}} ; \quad \Delta w_{\mathrm{i}} \equiv w_{\mathrm{i}}-w_{\mathrm{ni}} ; \quad\right\lrcorner c_{\mathrm{vi}} \equiv c_{\mathrm{vi}}-c_{\mathrm{ni}}$;

$$
\lrcorner c_{\mathrm{wi}} \equiv c_{\mathrm{wi}}-c_{\mathrm{ni}} ; \quad \Delta c_{\mathrm{i}} \equiv c_{\mathrm{i}}-c_{\mathrm{ni}} ; \quad \Delta p_{\mathrm{i}} \equiv p_{\mathrm{i}}-p_{\mathrm{n}},
$$

und erhalten aus (3.3), (3.4):

$$
\Delta v_{\mathrm{i}}=2 \alpha_{\mathrm{i}} \Delta c_{\mathrm{vi}} ; \quad \Delta w_{\mathrm{i}}=2 \alpha_{\mathrm{i}} \Delta_{c_{\mathrm{wi}}},
$$

und aus (2.12):

$$
\begin{aligned}
& 2 a_{\mathrm{i}} \Delta c_{\mathrm{i}}=\Delta v_{\mathrm{i}}+\Delta w_{\mathrm{i}} ; \quad \Delta c_{\mathrm{i}}=\Delta c_{\mathrm{vi}}+\Delta c_{\mathrm{wi}} ; \\
& 2 u_{\mathrm{i}}=\Delta v_{\mathrm{i}}-\Delta w_{\mathrm{i}} ; \quad u_{\mathrm{i}}=a_{\mathrm{i}}\left(\Delta c_{\mathrm{vi}}-\Delta c_{\mathrm{wi}}\right) .
\end{aligned}
$$

Mit den Formeln für $\Delta c_{\mathrm{i}}$ und $u_{\mathrm{i}}$ sind die Abweichungen von $c_{\mathrm{i}}$ und $u_{\mathrm{i}}$ von den Normalwerten $c_{\mathrm{ni}}$, 0 dargestellt durch die Abweichungen $\Delta c_{\mathrm{vi}}, \Delta c_{\mathrm{wi}}$ der Schallgeschwindigkeiten $c_{\mathrm{vi}}, c_{\mathrm{wi}}$ vom Normalwert $c_{\mathrm{ni}}$, welche auftreten würden, wenn einerseits die $v$-Welle allein in ein ungestörtes Gebiet hineinliefe, und andererseits die $w$-Welle allein in ein ungestörtes Gebiet 
hineinliefe. Aus gegebenem $\alpha_{\mathrm{i}}, g_{\mathrm{i}}, v_{\mathrm{i}}, w_{\mathrm{i}}$ und fest gewähltem $p_{\mathrm{n}}$ können diese $\Delta$-Größen leicht berechnet werden. Statt durch $v_{\mathrm{i}}, w_{\mathrm{i}}$ kann man den physikalischen Zustand also durch $\Delta c_{\mathrm{vi}}, \Delta c_{\mathrm{wi}}$ beschreiben, was oft bequemer ist.

In der fortschreitenden Welle ist entweder $A c_{\mathrm{vi}}=0$ oder $\Delta c_{\text {wi }}=0$; also hat in der fortschreitenden $v$-Welle die Strömungsgeschwindigkeit $u_{\mathrm{i}}$ das Vorzeichen von $\Delta c_{\mathrm{vi}}$, in der fortschreitenden $w$-Welle hat sie das umgekehrte Vorzeichen von $\Delta c_{w i}$. Die Gl. (3.2) erlaubt, zu $c_{\mathrm{vi}}$ den Druck $p_{\mathrm{vi}}$ zu berechnen, der in einer fortschreitenden Welle zum vorgegebenen $v_{\mathrm{i}}$ gehören würde:

$$
p_{\mathrm{vi}}=g_{\mathrm{i}} c_{\mathrm{vi}}{ }^{a_{\mathrm{i}}+2} \text {. }
$$

Ebenso kann man zu $c_{\text {wi }}$ den Druck $p_{\text {wi }}$ der zugehörigen fortschreitenden Welle berechnen:

$$
p_{\mathrm{wi}}=g_{\mathrm{i}} c_{\mathrm{wi}}{ }^{{ }^{{ }_{\mathrm{i}}+2}}{ }^{2} .
$$

\section{§4. Anwendung auf Reflexionsvorgänge und Rohrströmung}

1. Reflexion an fester Wand

Die Wand soll senkrecht zur Wanderrichtung der Wellen stehen; wir beschränken uns ja in dieser Arbeit auf ebene Wellen. An der Wand ist ständig $u=0$; den Index i können wir hier weglassen, an der Wand soll sich ein Gas von gegebener Adiabatenkonstante $g$ befinden. Aus (3.7) folgt an der Wand:

$$
\Delta c_{\mathrm{v}}=\Delta c_{\mathrm{w}} ;
$$

d. h. wegen (3.6): Aus jedem anlaufenden $v$-Wert wird ein ebenso großer zurücklaufender $w$-Wert, und umgekehrt. Außerdem ist an der Wand:

$$
\Delta c_{\mathrm{W}}=2 \Delta c_{\mathrm{v}}=2 \Delta c_{\mathrm{w}} ;
$$

der Index W soll andeuten, daß dieser $\Delta c$-Wert für den Zustand an der Wand gilt.

Charakterisiert man die anlaufende und die zurücklaufende Welle durch die Werte, welche ihnen zukommen, wenn sie einzeln in ungestörtem Gebiet laufen, wenn sie also fortschreitende Wellen sind, so kann man wegen (4.1) sagen: das $\Delta c$ der anlaufenden fortschreitenden Welle ist gleich dem $\Delta c$ der reflektierten fortschreitenden Welle. Die Wellenamplitude wird also durch die Wellenreflexion nicht geändert. Da in der fortschreitenden Welle entweder $\Delta c_{\mathrm{v}}$ oder $\Delta c_{\mathrm{w}}$ gleich Null ist, so kann man aus (3.7) schließen: die Strömungsgeschwindigkeit in der anlaufenden fort- schreitenden Welle ist entgegengesetzt gleich der Strömungsgeschwindigkeit in der reflektierten fortschreitenden Welle.

Weil nach (4.1) an der Wand $\Delta c_{\mathrm{v}}$ und $\Delta c_{\mathrm{W}}$ dasselbe Vorzeichen haben, so gilt: Überdruck wird als Überdruck reflektiert, Unterdruck als Unterdruck.

Für den Druck $p_{W}$ an der Wand ist:

$$
\begin{aligned}
\frac{p_{\mathrm{W}}}{p_{\mathrm{n}}} & =\left(\begin{array}{c}
c_{\mathrm{W}} \\
c_{\mathrm{n}}
\end{array}\right)^{a+2}=\left(1+\frac{\Delta c_{\mathrm{W}}}{c_{\mathrm{n}}}\right)^{a+2}=\left(1+\frac{2 \Delta c_{\mathrm{v}}}{c_{\mathrm{n}}}\right)^{a+2} \\
& =\left(\frac{2 c_{\mathrm{v}}}{c_{\mathrm{n}}}-1\right)^{a+2}=\left(2\left(\frac{p_{\mathrm{v}}}{p_{\mathrm{n}}}\right)^{1 /(a+2)}-1\right)^{a+2}
\end{aligned}
$$

also auch:

$$
\frac{\Delta p_{\mathrm{W}}}{p_{\mathrm{n}}}=\left[2\left(1+\frac{\Delta p_{\mathrm{v}}}{p_{\mathrm{n}}}\right)^{1 /(a+2)}-1\right]^{a+2}-1 .
$$

Der Ausdruck rechts ist für nicht verschwindendes $\Delta p_{\mathrm{v}}$ immer $>\frac{2 \Delta p_{\mathrm{v}}}{p_{\mathrm{n}}} ;$ im Grenzfall der Akustik wird:

$$
\Delta p_{\mathrm{W}} \approx 2 \Delta p_{\mathrm{v}},
$$

weil dann $\Delta p_{\mathrm{v}} \ll p_{\mathrm{n}}$ ist. Wegen $\Delta c_{\mathrm{v}}=\Delta c_{\mathrm{w}}$ an der Wand, kann man in den Gln. (4.3) bis (4.5) den Index $\mathrm{v}$ durch den Index w ersetzen.

\section{Reflexion am offenen Ende eines Rohres}

Die Größen, welche sich auf den Zustand am offenen Ende beziehen, bezeichnen wir mit dem Index E. Solange die Strömungsgeschwindigkeit dort kleiner als die Schallgeschwindigkeit ist, solange also $u_{\mathrm{E}}<c_{\mathrm{E}}$, können wir annehmen, daß der Druck $p_{\mathrm{E}}$ am offenen Ende gleich dem ungestörten Außendruck ist. Auch die Adiabatenkonstante $g$ können wir gleich derjenigen der Umgebung setzen. Den „Normaldruck“ $p_{\mathrm{n}}$, über den wir noch verfügen können, wählen wir hier gleich $p_{\mathrm{E}}$. Also gilt wegen (3.7):

$\left.p_{\mathrm{E}}=p_{\mathrm{n}} ; \quad \Delta p_{\mathrm{E}}=0 ; \quad \Delta c_{\mathrm{E}}=0=\Delta c_{\mathrm{v}}+\right\lrcorner c_{\mathrm{w}}$.

$\Delta c_{\mathrm{v}}$ und $\Delta c_{\mathrm{w}}$ haben am offenen Ende umgekehrtes Vorzeichen; aus einer anlaufenden Überdruckwelle wird also eine zurücklaufende Unterdruckwelle, und umgekehrt. Außerdem ist:

$$
\left.u_{\mathrm{E}}=2 a\right\lrcorner c_{\mathrm{v}}=-2 a \Delta c_{\mathrm{w}} .
$$

Das bedeutet: läuft Überdruck an, so geht die Strömung aus dem Rohr hinaus; läuft Unterdruck an, so übt das Rohr eine Saugwirkung aus. 
Deutet man die anlaufenden $v$ - bzw. $w$-Werte als fortschreitende Wellen, so gilt: das $\Delta c$ der anlaufenden fortschreitenden Welle ist entgegengesetzt gleich dem $\Delta c$ der reflektierten fortschreitenden Welle; die Strömungsgeschwindigkeit der anlaufenden fortschreitenden Welle ist gleich der Strömungsgeschwindigkeit in der reflektierten fortschreitenden Welle.

In den Drucken der fortschreitenden Wellen geschrieben, lauten die Gln. (4.6), (4.7):

$$
\begin{gathered}
\left(\begin{array}{c}
p_{\mathrm{v}} \\
p_{\mathrm{n}}
\end{array}\right)^{1 /(a+2)}+\left(\begin{array}{c}
p_{\mathrm{w}} \\
p_{\mathrm{n}}
\end{array}\right)^{1 /(a+2)}=2 \\
u_{\mathrm{E}}=2 \alpha\left[\left(\frac{p_{\mathrm{v}}}{p_{\mathrm{n}}}\right)^{1 /(a+2)}-1\right]=2 \alpha\left[1-\left(\frac{p_{\mathrm{w}}}{p_{\mathrm{n}}}\right)^{1 /(a+2)}\right] .
\end{gathered}
$$

Eine reflektierte Welle gibt es nur, solange $\left|u_{\mathrm{E}}\right| \lesssim c_{\mathrm{E}}$ ist; wir nehmen dabei an, daß auch für $\left|u_{\mathrm{E}}\right| \approx c_{\mathrm{n}}$ noch die Randbedingung $p_{\mathrm{E}}=p_{\mathrm{n}}$ wenigstens annähernd erfüllt ist. Wenn das Rohrende etwa rechts liegt und die anlaufende Welle von links kommt, also eine $v$-Welle ist, so muß wegen (3.7) für die anlaufende Welle gelten:

$$
\Delta c_{\mathrm{v}} \leq \frac{c_{\mathrm{n}}}{2 a}, \quad 2 a\left[\left(\frac{p_{\mathrm{v}}}{p_{\mathrm{n}}}\right)^{1 /(a+2)}-1\right] \leq 1
$$

wenn es eine zurücklaufende Welle geben soll. Allgemein darf das $\Delta c$ der anlaufenden Welle, die als fortschreitende Welle gedacht ist, nicht größer als

$$
\frac{c_{\mathrm{n}}}{2 \varkappa}=\frac{(*-1)}{4} c_{\mathrm{n}}
$$

sein. Für Luft ist das rund $1 / 10 c_{\mathrm{n}} ; p_{\mathrm{v}}$ wäre dann rund $1,94 \cdot p_{\mathrm{n}}$; bei äußerem Atmosphärendruck darf die anlaufende fortschreitende Welle nicht mehr als 0,94 at Überdruck haben, sonst tritt Überschall-Ausströmen aus dem Rohr auf, und die über 0,94 atü liegenden $v$-Werte werden nicht mehr reflektiert, sondern geben nur aus dem Rohr hinauslaufende Wellen.

3. Reflexion an der Grenzezweier Gebiete mit verschiedener Adiabaten-

$$
\text { konstante }
$$

Solche Gebiete gibt es in Verbrennungsmotoren, wo das Verbrannte eine andere Adiabatenkonstante hat als das Unverbrannte. Wir unterscheiden die beiden Gebiete durch die Indizes 1, 2. An der Grenze müssen Druck und Strömungsgeschwindigkeit stetig sein. Wegen der Stetigkeit von $u$ gilt nach (3.7):

$$
\left.a_{1}\left(\Delta c_{\mathrm{v} 1}-\Delta c_{\mathrm{w}_{1}}\right)=a_{2}\left(\Delta c_{\mathrm{v} 2}-\right\lrcorner c_{\mathrm{w} 2}\right) .
$$

Die Stetigkeit von $p$ verlangt:

$$
\frac{p_{1}}{p_{\mathrm{n}}}=\frac{p_{2}}{p_{\mathrm{n}}}=\left(\begin{array}{c}
c_{1} \\
c_{\mathrm{n}_{1}}
\end{array}\right)^{a_{1}+2}=\left(\begin{array}{c}
c_{2} \\
c_{\mathrm{n}_{2}}
\end{array}\right)^{a_{2}+2}
$$

das bedeutet:

$1+\frac{\Delta c_{\mathrm{v}_{1}}}{c_{\mathrm{n}_{1}}}+\frac{\Delta c_{\mathrm{w}_{1}}}{c_{\mathrm{n}_{1}}}=\left(1+\frac{\Delta c_{\mathrm{v} 2}}{c_{\mathrm{n} 2}}+\frac{\Delta c_{\mathrm{w} 2}}{c_{\mathrm{n} 2}}\right)^{\left(\alpha_{2}+2\right) /\left(a_{1}+2\right)}$.

In den Drucken der fortschreitenden Wellen geschrieben, lauten die Gln. (4.11), (4.13):

$$
\begin{aligned}
& \left(\frac{p_{\mathrm{v}_{1}}}{p_{\mathrm{n}}}\right)^{1 /\left(a_{1}+2\right)}-\left(\frac{p_{\mathrm{w}_{1}}}{p_{\mathrm{n}}}\right)^{1 /\left(a_{1}+2\right)} \\
& =\frac{a_{2}}{a_{1}} \frac{c_{\mathrm{n}_{2}}}{c_{\mathrm{n}_{1}}}\left[\left(\frac{p_{\mathrm{v}_{2}}}{p_{\mathrm{n}}}\right)^{1 /\left(a_{2}+2\right)}-\left(\frac{p_{\mathrm{w}_{2}}}{p_{\mathrm{n}}}\right)^{1 /\left(a_{2}+2\right)}\right] \\
& \left(\frac{p_{\mathrm{v}_{1}}}{p_{\mathrm{n}}}\right)^{1 /\left(a_{1}+2\right)}+\left(\frac{p_{\mathrm{w}_{1}}}{p_{\mathrm{n}}}\right)^{1 /\left(a_{1}+2\right)}-1 \\
& =\left[\left(\frac{p_{\mathrm{v} 2}}{p_{\mathrm{n}}}\right)^{1 /\left(a_{2}+2\right)}+\left(\frac{p_{\mathrm{w}_{2}}}{p_{\mathrm{n}}}\right)^{1 /\left(a_{2}+2\right)}-1\right]^{\left(a_{2}+2\right) /\left(a_{1}+2\right)}
\end{aligned}
$$

$c_{\mathrm{n}_{2}} / c_{\mathrm{n}_{1}}$ kann man nach (3.1) auch umschreiben:

$$
c_{\mathrm{n}_{2}}=\frac{g_{1}{ }^{1 /\left(a_{1}+2\right)}}{c_{\mathrm{n}_{1}}} \frac{g_{2}^{1 /\left(a_{2}+2\right)}}{\text {, }}
$$

also durch die Adiabatenkonstanten und Freiheitsgrade ausdrücken.

\section{Beliebige ebene Welle}

Druck und Strömungsgeschwindigkeit einer beliebigen ebenen Welle kann man mit Hilfe von (3.7) ebenfalls durch die Drucke darstellen, welche in den zugeordneten fortschreitenden Wellen herrschen würden, also in den Wellen, welche aus der ebenen Welle durch das Auseinanderlaufen der $v$ - und $w$-Welle hervorgehen, wenn die ebene Welle sich frei ausbreiten kann. So ist:

$$
\begin{gathered}
\frac{p}{p_{\mathrm{n}}}=\left[\left(\frac{p_{\mathrm{v}}}{p_{\mathrm{n}}}\right)^{1 /(a+2)}+\left(\frac{p_{\mathrm{w}}}{p_{\mathrm{n}}}\right)^{1 /(a+2)}-1\right]^{a+2} ; \\
u \\
c_{\mathrm{n}}=a\left[\left(\frac{p_{\mathrm{v}}}{p_{\mathrm{n}}}\right)^{1 /(a+2)}-\left(\begin{array}{l}
p_{\mathrm{w}} \\
p_{\mathrm{n}}
\end{array}\right)^{1 /(a+2)}\right]
\end{gathered}
$$


5. Strömung in einem Rohr mit einem geschlossenen und einem offenen Ende

Das offene Ende sei rechts, das geschlossene links. Wenn Überdruck gegen das offene Ende anläuft, wird nach den Überlegungen in $\S 4.2$ Unterdruck zurücklaufen, solange $\Delta c_{\mathrm{v}} \leq c_{\mathrm{n}} / 2 \alpha$ ist; wegen (4.6) gilt für den zurücklaufenden Unterdruck: $\Delta c_{\mathrm{w}} \geq-c_{\mathrm{n}} / 2 \alpha$.

Wir berechnen jetzt den größten Unterdruck, der durch die zurücklaufende Welle am geschlossenen Ende erzeugt werden kann. Dort ist $\boldsymbol{u}_{\mathrm{W}}=0$.

a) Wenn im Rohr nur Gas der gleichen Adiabatenkonstante vorhanden ist, so gilt nach (4.3) und (4.2):

$$
\frac{p_{\mathrm{W}}}{p_{\mathrm{n}}} \geq\left(1-\frac{1}{a}\right)^{a+2} \text {. }
$$

Das Gleichheitszeichen gibt den kleinsten Druck am geschlossenen Ende; ihm entspricht ein größter Unterdruck von:

$$
\left|\Delta p_{\mathrm{W}}\right|_{\max }=p_{\mathrm{n}}\left[1-\left(1-\frac{1}{a}\right)^{a+2}\right] .
$$

Für $\varkappa=1,40$, was annähernd Luft bei Normalbedingungen entspricht, ist $\alpha=5$ und

$$
p_{\text {Wmin }}=0,21 p_{\mathrm{n}} ;\left|\Delta p_{\mathrm{W}}\right|_{\max }=0,79 p_{\mathrm{n}} .
$$

Die rechte Seite der Ungleichung (4.19) hat für $\alpha=1$ den Wert Null und wächst mit wachsendem $\alpha$, für großes $\alpha$ strebt die Funktion gegen den Wert $1 / e=0,37$.

b) Wird das Rohr als Motor betrieben, so gibt es im allgemeinen zwei Gasgebiete verschiedener Adiabatenkonstante darin. Am offenen Ende sei ein Gasgebiet mit der Konstanten $g_{1}$, am geschlossenen Ende ein Gasgebiet mit $g_{2}$. Für den stärksten vom offenen Ende zurücklaufenden Unterdruck ist: $\Delta c_{\mathrm{w}_{1}}=-c_{\mathrm{n}_{1}} / 2 \alpha_{1}$. An der Grenze der Gebiete 1, 2 gelten die Gln. (4.11), (4.13) mit $\Delta c_{\mathrm{v} 2}=0$. Aus (4.11) folgt so:

$$
J c_{\mathrm{v} 1}=\Delta c_{\mathrm{w} 1}-{ }_{a_{1}}^{a_{2}} \Delta c_{\mathrm{w} 2} .
$$

Das gibt aus (4.13) für den stärksten zurücklaufenden Unterdruck:

$\left(1+\frac{J c_{\mathrm{w}_{2}}}{c_{\mathrm{n}_{2}}}\right)^{\left(a_{2}+2\right) /\left(a_{1}+2\right)}=1-\frac{1}{a_{1}}-\frac{a_{2}}{a_{1}} \frac{J c_{\mathrm{w}_{2}}}{c_{\mathrm{n}_{1}}}$.

Aus dieser Gleichung ist $\Delta c_{\mathrm{w}_{2}}$ zu berechnen, dann ergibt sich für den stärksten Unterdruck am geschlossenen Ende aus (4.3):

$$
\left(\begin{array}{c}
p_{\mathrm{W}} \\
p_{\mathrm{n}}
\end{array}\right)_{\min }=\left(1+\frac{2\lrcorner c_{\mathrm{w}_{2}}}{c_{\mathrm{n} 2}}\right)^{a_{2}+2},
$$

wobei $\Delta c_{\mathrm{w} 2}$ aus (4.21) einzusetzen ist. Wenn $\alpha_{1}=\alpha_{2}$ $\equiv \alpha$, so wird aus (4.22):

$$
\left(\frac{p_{\mathrm{W}}}{p_{\mathrm{n}}}\right)_{\min }=\left(1-\frac{2}{\left(1+c_{\mathrm{n}_{2}} / c_{\mathrm{n}_{1}}\right) a}\right)^{a+2} .
$$

Um ein Beispiel für Verhältnisse zu geben, wie sie in Motoren vorkommen, nehmen wir an, es sei: $c_{\mathrm{n}_{1}}=2 c_{\mathrm{n}_{2}} ; c_{\mathrm{n}_{1}}$ entspricht verbranntem Gas, $c_{\mathrm{n} 2}$ dem Frischgas; $\alpha$ setzen wir gleich 5 . Dann wird $\left(p_{\mathrm{W}} / p_{\mathrm{n}}\right)_{\min }$ $=0,11$.

Wir schließen einige qualitative Bemerkungen ${ }^{3}$ an über die Strömung in einem einseitig offenen Rohr, wenn in der geschlossenen Wand Ventile angebracht

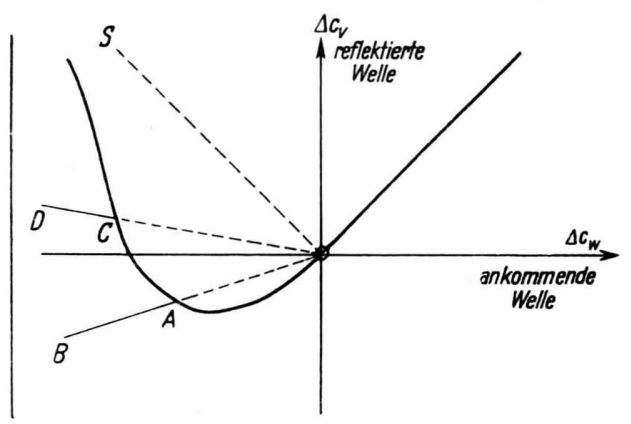

Abb. 1. Reflexion an der mit Luftklappen versehenen Stirnwand eines einseitig offenen Rohres; $\Delta c_{\mathrm{v}}$ der reflektierten Welle in Abhängigkeit von $\Delta c_{\mathrm{w}}$ der ankommenden Welle, schematisch. Zeichenerklärung im Text.

sind, die sich öffnen, sobald im Rohr Unterdruck herrscht gegenüber dem Druck auf der Außenseite dieser Wand; wir nennen die Wand jetzt „Stirnwand“. Sie liege wieder links, das offene Ende rechts. Wir nehmen an, daß außen an der Stirnwand der Druck $p_{\mathrm{n}}$ herrscht, was bei einem ruhenden Motor zutrifft. Wird das Rohr als Antrieb verwendet, so ist der Druck außen an der Stirnwand größer als $p_{\mathrm{n}}$, da wir den Druck am offenen Ende des Rohres weiter $p_{n}$ nennen wollen.

Wenn die Ventile aufgehen, wird eine ziemlich regellose Strömung und Druckverteilung in nächster Nähe der Stirnwand im Rohr entstehen, von der wir annehmen können, daß sie in einiger Entfernung von der Stirnwand sich wieder zu einer einigermaßen geregelten Strömung, einer Überdruck- und Unterdruckwelle zusammensetzt, so daß auch die reflektierte Welle durch ein $\Delta c$, hier durch $\Delta c_{\mathrm{V}}$, charak-

3 Eine quantitative Theorie der Vorgänge in einem solchen Rohr ist von uns in den oben zitierten Arbeiten gegeben worden. 
terisiert werden kann. Diese Annahme ist durch Beobachtungen ausreichend bestätigt. In Abb. 1 stellen wir $\Delta c_{\mathrm{r}}$ in Abhängigkeit von $\Delta c_{\mathrm{w}}$ schematisch dar. Im Sinn der Bezeichnung von Gl. (4.21) müßten wir $A c_{\mathrm{v} 2}$ und $\Delta c_{\mathrm{w} 2}$ schreiben; wir lassen aber den Index 2 jetzt weg. Solange $\Delta c_{\mathrm{w}}>0$, kommt Überdruck an, die Ventile bleiben geschlossen, und es gilt: $\Delta c_{\mathrm{v}}=\Delta c_{\mathrm{w}}$. Das gibt die gerade Linie nach rechts oben in Abb. 1 .

Sobald $\Delta c_{\mathrm{w}}<0$ ist, gehen die Ventile auf, bei kleinem $\left|\Delta c_{\mathrm{w}}\right|$ nur wenig. Die Wand wirkt als noch fast geschlossen; so daß immer noch $\Delta c_{\mathrm{v}} \approx \Delta c_{\mathrm{w}}$ ist. Dem entspricht die annähernd geradlinige Fortsetzung der Kurve links von 0 nach unten in Abb. 1 . Bei sehr großem negativen $\Delta c_{\mathrm{w}}$ wird Überdruck reflektiert, wenn die Ventile weit genug aufgehen können. Lassen sie sich so weit öffnen, daß fast nichts mehr an reflektierender Wand da ist, dann wird $\Delta c_{\mathrm{v}} \approx-\Delta c_{\mathrm{w}}$. Die Kurve in Abb. 1 muß sich also der gestrichelten Geraden OS nähern. Der reflektierte Unterdruck nimmt demnach mit zunehmendem ankommendem Unterdruck nur bis zu einem größten Wert zu und dann wieder $\mathrm{ab}$ und geht schließlich in reflektierten Überdruck über. Unter plausiblen Annahmen ergibt sich für geöffnete Ventile, wie in den oben zitierten Arbeiten über das Schmidt-Rohr gezeigt wurde ${ }^{1}$ :

$$
\lrcorner c_{\mathrm{v}}=\lambda(\eta)\right\lrcorner c_{\mathrm{w}} ;
$$

$\eta$ ist das Verhältnis von reflektierendem Wandquerschnitt zum gesamten Wandquerschnitt; $\lambda$ ist eine Funktion von $\eta$ allein. $\eta=1$ bedeutet geschlossene Ventile, $\eta=0$ heißt, daß gar keine Stirnwand da ist. Es ist $\lambda(1)=1 ; \lambda(0)=-1$. Wenn die Ventile nur so weit aufgehen können, daß auch bei größter Öffnung noch Unterdruck reflektiert wird (Punkt A in Abb. 1), so ist $\eta=\eta_{\min }$, und $\lambda(\eta)=\lambda\left(\eta_{\min }\right)$ ein fester Wert, auf $\operatorname{dem} \lambda$ stehen bleibt, wenn noch stärkerer Unterdruck ankommt. Bei weiter wachsendem Unterdruck besteht also wieder (annähernd) Proportionalität zwischen $\Delta c_{\mathrm{v}}$ und $\Delta c_{\mathrm{w}}$. Die Kurve wird in diesem Fall bei zunehmendem Unterdruck von 0 aus nur bis zum Punkt A durchlaufen. Von dort an stellt die von 0 ausstrahlende Gerade $\mathrm{AB}$ den Zusammenhang zwischen $\Delta c_{\mathrm{v}}$ und $\Delta c_{\mathrm{w}}$ dar. Sinngemäß dasselbe gilt, wenn die Ventile so weit aufgehen können, daß Überdruck reflektiert wird (Punkt $\mathrm{C}$ auf der Kurve). In diesem Fall würde die Kurve von 0 über A bis C durchlaufen und dann sich die Gerade CD daran schließen. Die Vertikale links in Abb. 1 gibt die Grenze an, bis zu der $\Delta c_{\mathrm{w}}$ nach Gl. (4.21) abnehmen kann.

\section{§. Entwicklung nach $\Delta c_{\mathrm{i}} / c_{\mathrm{ni}}$. Die reduzierten Drucke}

Die in den Gleichungen der Akustik verwendete Näherung bedeutet Entwicklung nach $\Delta p / p_{\mathrm{n}}$ und Beibehaltung der Glieder erster Ordnung. Für $p_{\mathrm{n}}=1$ at kann $\Delta p$ also nicht über etwa 0,1 at hinausgehen.

Die in der Praxis vorkommenden Druckstörungen bei Rohrströmung oder Motorenvorgängen sind weit größer, gehen aber häufig nicht über etwa $\Delta p / \Delta p_{\mathrm{n}} \approx 2$ hinaus. Die akustische Näherung ist hier unzureichend, ja sie kann zu unsinnigen Ergebnissen führen wie im Fall der Reflexion am offenen Rohrende, wo sich für $\Delta p / p_{\mathrm{n}}>1$ negativer reflektierter Druck ergeben würde. In diesem Bereich steigt $\Delta c / c_{\mathrm{n}}$ nicht wesentlich über 0,15; deshalb ist es hier zweckmäßig, nach den Größen $\Delta c / c_{\mathrm{n}}$ zu entwickeln. Man erfaßt so durch einfache Formeln die genannten praktisch wichtigen Strömungsvorgänge; dieses Näherungsverfahren reicht weit über den akustischen Bereich hinaus, dessen Ergebnisse den Grenzfall $\Delta p / p_{\mathrm{n}} \ll 1$ unseres Näherungsverfahrens bilden.

Bevor wir das Verfahren allgemein besprechen, geben wir ein Beispiel. Eine $v$-Welle laufe gegen die feste Wand $\mathrm{W}$ an; es sei $p_{\mathrm{v}}=2$ at; $p_{\mathrm{n}}=1$ at, $\alpha=5$. Das bedeutet:

$$
\frac{c_{\mathrm{v}}}{c_{\mathrm{n}}}=\left(\frac{p_{\mathrm{v}}}{p_{\mathrm{n}}}\right)^{1 /(a+2)}=2^{1 / 7}=1,10 ; \quad \begin{gathered}
\Delta c_{\mathrm{v}} \\
c_{\mathrm{n}}
\end{gathered}=0,10 .
$$

Wegen (4.2) ist allgemein:

$$
\frac{\Delta c_{\mathrm{W}}}{c_{\mathrm{n}}}=2 \frac{\Delta c_{\mathrm{v}}}{c_{\mathrm{n}}}
$$

also:

$$
\begin{gathered}
c_{\mathrm{W}}=1+\frac{2 \Delta c_{\mathrm{v}}}{c_{\mathrm{n}}}=\left(1+\frac{\Delta c_{\mathrm{v}}}{c_{\mathrm{n}}}\right)^{2}-\left(\begin{array}{c}
\Delta c_{\mathrm{v}} \\
c_{\mathrm{n}}
\end{array}\right)^{2} \\
=\left(\begin{array}{c}
c_{\mathrm{v}} \\
c_{\mathrm{n}}
\end{array}\right)^{2}\left(1-\left(\begin{array}{c}
\Delta c_{\mathrm{v}} \\
c_{\mathrm{v}}
\end{array}\right)^{2}\right) \\
\frac{p_{\mathrm{W}}}{p_{\mathrm{n}}}=\left(\frac{p_{\mathrm{v}}}{p_{\mathrm{n}}}\right)^{2}\left(1-\left(\begin{array}{c}
\Delta c_{\mathrm{v}} \\
c_{\mathrm{v}}
\end{array}\right)^{2}\right)^{a+2} .
\end{gathered}
$$

(5.1), (5.2) gelten streng und allgemein. In unserem Beispiel ist $\left(\Delta c_{\mathrm{v}} / c_{\mathrm{v}}\right)^{2}=10^{-2} / 1,21=8,3 \cdot 10^{-3} \ll 1$. Hier kann man also die zweite Klammer in (5.2) rechts durch Eins ersetzen. Noch für $c_{\mathrm{v}} / c_{\mathrm{n}}=1,20$, was $p_{\mathrm{v}} / p_{\mathrm{n}}=3,60$ bedeutet, ist der Fehler kleiner als 2\%, wenn man die zweite Klammer rechts in (5.2) nach $\left(\Delta c_{\mathrm{v}} / c_{\mathrm{v}}\right)^{2}$ nur bis zur ersten Potenz dieser Größe entwickelt. Es wird häufig für die Anwendungen 
genügen, wenn man die Formeln bis zu quadratischen, höchstens kubischen Gliedern in $\Delta c / c_{\mathrm{n}}$ einschließlich entwickelt.

Die Form der Gl. (5.2) legt nahe, an Stelle der Drucke ihr Verhältnis zum Normaldruck $p_{\text {n }}$ einzuführen, für das wir die Bezeichnung ,reduzierter Druck“ verwenden wollen. Wir schreiben diese Größen mit $P$ und definieren also:

$P \equiv \frac{p}{p_{\mathrm{n}}} ; \quad P_{\mathrm{W}} \equiv \frac{p_{\mathrm{W}}}{p_{\mathrm{n}}} ; \quad P_{\mathrm{v}} \equiv \frac{p_{\mathrm{v}}}{p_{\mathrm{n}}} ; \quad P_{\mathrm{w}} \equiv \frac{p_{\mathrm{w}}}{p_{\mathrm{n}}}$.

Aus (5.2) wird so, wenn wir in der besprochenen Weise entwickeln:

$$
P_{\mathrm{W}}=P_{\mathrm{v}}{ }^{2}\left(1-(\alpha+2)\left(\frac{\Delta c_{\mathrm{v}}}{c_{\mathrm{v}}}\right)^{2}\right) .
$$

In erster Näherung wäre dies: $P_{\mathrm{W}} \cdot P_{\mathrm{v}}{ }^{-2}=1$. Halten wir die Gl. (4.2) daneben in der Form:

$$
\frac{\lrcorner c_{\mathrm{W}}}{c_{\mathrm{n}}}-\frac{2 \Delta c_{\mathrm{v}}}{c_{\mathrm{n}}}=0,
$$

so sehen wir, daß aus der in den $\Delta c / c_{\mathrm{n}}$ linearen Beziehung (4.2) eine Produktbeziehung in den $P$ geworden ist, wobei die Faktoren vor den $\Delta c / c_{\mathrm{n}}$ zu den Exponenten der $P$ werden.

Das ist ein Spezialfall eines allgemeinen Zusammenhangs. Wenn für hinreichend kleine Größen $\Delta c_{\mathrm{i}} / c_{\mathrm{ni}}$ eine lineare Beziehung mit den Koeffizienten $\beta_{\mathrm{i}}$ besteht:

$$
\sum_{\mathrm{i}} \beta_{\mathrm{i}} \frac{\Delta c_{\mathrm{i}}}{c_{\mathrm{ni}}}=0,
$$

so folgt für die durch:

$$
P_{\mathrm{i}} \equiv \frac{p_{\mathrm{i}}}{p_{\mathrm{n}}}=\left(\frac{c_{\mathrm{i}}}{c_{\mathrm{ni}}}\right)^{a+2}
$$

definierten Größen $P_{\mathrm{i}}$ aus (5.6) unter Vernachlässigung von Gliedern der Ordnung $\left(\Delta c_{\mathrm{i}} / c_{\mathrm{ni}}\right)^{2}$ :

$$
\prod_{\mathrm{i}} P_{\mathrm{i}}^{\beta_{\mathrm{i}}}=1 \text {. }
$$

Die Gleichung ist durch Reihenentwicklung leicht zu beweisen, wobei wir die in (5.8) nicht angeschriebenen Glieder höherer Ordnung in $\Delta c_{\mathrm{i}} / c_{\mathrm{ni}}{ }^{2}$ gleich mitbekommen. Wir setzen voraus, daß alle $\Delta c_{\mathrm{i}} / c_{\mathrm{ni}}$ zum gleichen $\alpha$ gehören, so daß wir an $\alpha$ keinen Index zu hängen brauchen. Es ist:

$$
P_{\mathrm{i}}^{\beta_{\mathrm{i}}}=\left(\begin{array}{l}
p_{\mathrm{i}} \\
p_{\mathrm{n}}
\end{array}\right)^{\beta_{\mathrm{i}}}=\left(1+\begin{array}{c}
\lrcorner c_{\mathrm{i}} \\
c_{\mathrm{ni}}
\end{array}\right)^{\beta_{\mathrm{i}}(n+2)}
$$

also:

$$
\begin{aligned}
& \beta_{\mathrm{i}} \log P_{\mathrm{i}} \approx \beta_{\mathrm{i}}(a+2)\left[\frac{\Delta c_{\mathrm{i}}}{c_{\mathrm{ni}}}-\frac{1}{2}\left(\frac{\Delta c_{\mathrm{i}}}{c_{\mathrm{ni}}}\right)^{2}+\frac{1}{3}\left(\frac{\Delta c_{\mathrm{i}}}{c_{\mathrm{ni}}}\right)^{3} \mid ;\right. \\
& \sum_{\mathrm{i}} \beta_{\mathrm{i}} \log P_{\mathrm{i}}=\log \left(\prod_{\mathrm{i}} P_{\mathrm{i}}^{\beta_{\mathrm{i}}}\right) \\
& \approx-\frac{(a+2)}{2} \sum_{\mathrm{i}} \beta_{\mathrm{i}}\left(\frac{\Delta c_{\mathrm{i}}}{c_{\mathrm{ni}}}\right)^{2}+\frac{(a+2)}{3} \sum_{\mathrm{i}} \beta_{\mathrm{i}}\left(\frac{\Delta c_{\mathrm{i}}}{c_{\mathrm{ni}}}\right)^{3}
\end{aligned}
$$

folgt:

$$
\begin{aligned}
\prod_{\mathrm{i}} P_{\mathrm{i}}^{\beta_{\mathrm{i}}} \approx 1-\frac{(\alpha+2)}{2} & \sum_{\mathrm{i}} \beta_{\mathrm{i}}\left(\frac{\Delta c_{\mathrm{i}}}{c_{\mathrm{ni}}}\right)^{2} \\
+ & \frac{(\alpha+2)}{3} \sum_{\mathrm{i}} \beta_{\mathrm{i}}\left(\frac{\Delta c_{\mathrm{i}}}{c_{\mathrm{ni}}}\right)^{3} .
\end{aligned}
$$

In erster Näherung kommt wirklich Gl. (5.8) heraus. Für die Reflexion an einer festen Wand gilt also:

$$
P_{\mathrm{W}} \approx P_{\mathrm{v}}{ }^{2}=P_{\mathrm{w}}^{2} \text {, }
$$

mit einer Ungenauigkeit von höchstens rund $(\alpha+2) \%$, wenn $\left|\Delta c_{\mathrm{v}} / c_{\mathrm{n}}\right|$ nicht größer als 0,1 ist.

Bei der Reflexion am offenen Ende gilt nach (4.6):

$$
\frac{\Delta c_{\mathrm{v}}}{c_{\mathrm{n}}}+\frac{\Delta c_{\mathrm{w}}}{c_{\mathrm{n}}}=0,
$$

woraus in unserer Näherung folgt:

$$
P_{\mathrm{w}} \approx \frac{1}{P_{\mathrm{v}}},
$$

mit einer Ungenauigkeit von höchstens rund $(\alpha+2) \%$, wenn $\left|\Delta c_{\mathrm{v}} / c_{\mathrm{n}}\right|$ nicht größer als 0,1 ist.

Das bedeutet z. B., daß für $p_{\mathrm{n}}=1$ at ein anlaufender Druck $p_{\mathrm{v}}$ von 2 at durch die Reflexion am offenen Ende in einen Druck $p_{\mathrm{w}}$ von $1 / 2$ at verwandelt wird. Die Akustik würde das unsinnige Ergebnis $p_{w}=0$ liefern.

Für die Darstellung des Druckes einer ebenen Welle durch die Drucke $p_{\mathrm{v}}, p_{\mathrm{w}}$ der entsprechenden fortschreitenden Wellen bekommt man aus (3.7):

$$
\frac{\Delta c}{c_{\mathrm{n}}}-\frac{\Delta c_{\mathrm{v}}}{c_{\mathrm{n}}}-\frac{\Delta c_{\mathrm{w}}}{c_{\mathrm{n}}}=0
$$

die Näherungsgleichung:

$$
P \approx P_{\mathrm{v}} P_{\mathrm{w}} .
$$

Auch die Reflexion an der Grenze von zwei Medien läßt sich in dieser einfachen Weise behandeln, wenn $\alpha$ in beiden Medien als praktisch gleich angesehen werden kann. Dann gelten die Gln. (4.11), (4.13) in der Form: 


$$
\begin{aligned}
\frac{J c_{\mathrm{v} 1}}{c_{\mathrm{n}_{1}}}-\frac{\Delta c_{\mathrm{w}_{1}}}{c_{\mathrm{n}_{1}}}=\left(\frac{\Delta c_{\mathrm{v} 2}}{c_{\mathrm{n}_{2}}}-\frac{\Delta c_{\mathrm{w}_{2}}}{c_{\mathrm{n}_{2}}}\right) \frac{c_{\mathrm{n}_{2}}}{c_{\mathrm{n}_{1}}} \\
\frac{\Delta c_{\mathrm{v}_{1}}}{c_{\mathrm{n}_{1}}}+\frac{\Delta c_{\mathrm{w}_{1}}}{c_{\mathrm{n}_{1}}}=\frac{\Delta c_{\mathrm{v}_{2}}}{c_{\mathrm{n}_{2}}}+\frac{\Delta c_{\mathrm{w}_{2}}}{c_{\mathrm{n}_{2}}} .
\end{aligned}
$$

Das gibt die zwei Näherungsgleichungen:

$$
\begin{aligned}
& \frac{P_{\mathrm{v} 1}}{P_{\mathrm{w}_{1}}} \approx\left(\frac{P_{\mathrm{v} 2}}{P_{\mathrm{w} 2}}\right)^{c_{\mathrm{n} 2} / c_{\mathrm{n} 1}} ; \\
& P_{\mathrm{v} 1} P_{\mathrm{w} 1} \approx P_{\mathrm{v} 2} P_{\mathrm{w} 2},
\end{aligned}
$$

aus denen z. B. $P_{\mathrm{v} 2}, P_{\mathrm{w} 2}$ berechnet werden können, wenn $P_{\mathrm{v} 1}, P_{\mathrm{w} 1}$ bekannt sind. Man sieht, daß die Gleichungen erheblich einfacher sind als die strengen Gln. (4.14), (4.15).

Auch die in $\$ 4.5$ angegebene Näherungsgleichung für die Reflexion an der Stirnwand eines Rohres mit Ventilen läßt sich nach dem hier gegebenen Verfahren leicht auf die reduzierten Drucke umschreiben. Aus $1 c_{\mathrm{v}}=\lambda(\eta) \Delta c_{\mathrm{w}}$ folgt näherungsweise:

$$
P_{\mathrm{v}} \approx P_{\mathrm{w}}^{\lambda(\eta)}
$$

Aus der Formel (3.7) kann man schließlich noch eine Näherungsformel für die Strömungsgeschwindigkeit $u$ gewinnen. Es ist nach (3.7):

$$
\frac{u}{a c_{\mathrm{n}}}=\frac{c_{\mathrm{v}}}{c_{\mathrm{n}}}-\frac{c_{\mathrm{w}}}{c_{\mathrm{n}}} \text {. }
$$

Da nun $c_{\mathrm{v}}, c_{\mathrm{w}}$ immer ziemlich nahe an Eins liegen, können wir entwickeln:

$$
\begin{aligned}
& \frac{u}{a c_{\mathrm{n}}}=e^{\lg c_{\mathrm{v}} / c_{\mathrm{n}}}-e^{\lg c_{\mathrm{w}} / c_{\mathrm{n}}} \\
& \approx 1+\lg \frac{c_{\mathrm{v}}}{c_{\mathrm{n}}}+\frac{1}{2}\left(\lg \frac{c_{\mathrm{v}}}{c_{\mathrm{n}}}\right)^{2}-1-\lg \frac{c_{\mathrm{w}}}{c_{\mathrm{n}}}-\frac{1}{2}\left(\lg \frac{c_{\mathrm{w}}}{c_{\mathrm{n}}}\right)^{2} \\
& =\left(\lg \frac{c_{\mathrm{v}}}{c_{\mathrm{n}}}-\lg \frac{c_{\mathrm{w}}}{c_{\mathrm{n}}}\right)\left[1+\frac{1}{2}\left(\lg \frac{c_{\mathrm{v}}}{c_{\mathrm{n}}}+\lg \frac{c_{\mathrm{w}}}{c_{\mathrm{n}}}\right)\right] \\
& =\frac{1}{a+2} \lg \left(\frac{P_{\mathrm{v}}}{P_{\mathrm{w}}}\right)\left[1+\frac{1}{2(a+2)} \lg \left(P_{\mathrm{v}} P_{\mathrm{w}}\right)\right] .
\end{aligned}
$$

Das gibt wegen (5.12):

$$
\frac{u}{c_{\mathrm{n}}} \approx \frac{a}{a+2} \lg \left(\frac{P_{\mathrm{v}}}{P_{\mathrm{w}}}\right)\left[1+\frac{1}{2(a+2)} \lg P\right] .
$$

In erster Näherung kann die eckige Klammer gleich Eins gesetzt werden.

Mit den hier entwickelten Näherungsmethoden können Wellenvorgänge in Motoren und Rohren mit maximalen Überdrucken von etwa 1,5 at ausreichend beschrieben werden, wie die Verfasser in den oben zitierten Arbeiten über das Schmidt-Rohr gezeigt haben.

\title{
27d-Variation der harten Komponente der kosmischen Strahlung und Magnetfeld der Erde zur Zeit eines Minimums der Sonnenaktivität
}

\author{
Von Herma Gheri \\ Aus dem Physikalischen Institut der Universität Innsbruck \\ (Z. Naturforschg. 6 a, 775-780 [1951]; eingegangen am 18. September 1951)
}

\begin{abstract}
An einem 23 Sonnenrotationen umfassenden Beobachtungsmaterial der kosmischen Strahlung wird gezeigt, daß nur ein geringer Anteil der 27-Tage-Variation durch erdmagnetische Einflüsse gedeutet werden kann. Nach der magnetischen Korrektur der Strahlungswerte zeigt die Häufigkeitsverteilung der Lage des Maximums im Gegensatz zur unkorrigierten Strahlung zwei den Störpegel deutlich überragende Spitzen: a) 12 Rotationen mit Maximum am 1.-6. Tag, b) 5 Rotationen mit Maximum am 9.-11. Tag. Während der Rotationen a), die hauptsächlich zur Zeit der magnetischen Wiederkehr beobachtet wurden, scheint die Emission der Wellenstrahlung der Sonne am Beginn des Rotationsintervalls verringert zu sein (erschlossen aus dem Verlauf der Bodentemperatur), so daß es plausibel erscheint, die Variation der Strahlung wähzend dieser Rotationen durch atmosphärische Einflüsse zu erklären. Eine Deutung der Variationen b) war nicht möglich.
\end{abstract}

A. Übersicht über die Ursachen

$$
\text { der } 27^{\text {d }}-\text { V ariation }
$$

$A^{\text {ls }}$ Ursache der $27^{\mathrm{d}}$-Variation der kosmischen StrahAlung (KS) kommen folgende Erscheinungen in Betracht:
1. Die Stellung der Erde im Magnetfeld der Sonne ändert sich periodisch infolge der Rotation der Sonne (Vallarta-Godart).

2. Aktive Zentren auf der Sonne, deren Lebensdauer größer ist als eine Sonnenrotation, können die KS auf folgende Weise beeinflussen: 